\title{
On the mixed problem for quasilinear partial functional differential equations with unbounded delay
}

\author{
by Tomasz CzŁapiński (Gdańsk)
}

\begin{abstract}
We consider the mixed problem for the quasilinear partial functional differential equation with unbounded delay

$$
D_{t} z(t, x)=\sum_{i=1}^{n} f_{i}\left(t, x, z_{(t, x)}\right) D_{x_{i}} z(t, x)+h\left(t, x, z_{(t, x)}\right),
$$

where $z_{(t, x)} \in \mathfrak{X}_{0}$ is defined by $z_{(t, x)}(\tau, s)=z(t+\tau, x+s),(\tau, s) \in(-\infty, 0] \times[0, r]$, and the phase space $\mathfrak{X}_{0}$ satisfies suitable axioms. Using the method of bicharacteristics and the fixed-point method we prove a theorem on the local existence and uniqueness of Carathéodory solutions of the mixed problem.
\end{abstract}

1. Introduction. Let $B=\mathbb{R}_{-} \times[0, r]$, where $r=\left(r_{1}, \ldots, r_{n}\right) \in$ $\mathbb{R}_{+}^{n}\left(\mathbb{R}_{+}=[0, \infty), \mathbb{R}_{-}=(-\infty, 0]\right)$. For a given function $z:(-\infty, a] \times$ $[-b, b+r] \rightarrow \mathbb{R}$, where $a>0, b=\left(b_{1}, \ldots, b_{n}\right), b_{i}>0, i=1, \ldots, n$, and a point $(t, x)=\left(t, x_{1}, \ldots, x_{n}\right) \in[0, a] \times[-b, b]$, we define $z_{(t, x)}: B \rightarrow \mathbb{R}$ by

$$
z_{(t, x)}(\tau, s)=z(t+\tau, x+s), \quad(\tau, s) \in B .
$$

Define $\partial_{0} E_{\bar{a}}=[0, \bar{a}] \times[-b, b+r] \backslash[0, \bar{a}] \times[-b, b), E_{\bar{a}}=[0, \bar{a}] \times[-b, b]$ and $E_{\bar{a}}^{*}=(-\infty, \bar{a}] \times[-b, b+r]$ for any $\bar{a} \in[0, a]$.

For given functions $\phi: E_{0}^{*} \cup \partial_{0} E_{a} \rightarrow \mathbb{R}, h: E_{a} \times \mathfrak{X}_{0} \rightarrow \mathbb{R}, f=$ $\left(f_{1}, \ldots, f_{n}\right): E_{a} \times \mathfrak{X}_{0} \rightarrow \mathbb{R}^{n}$, where the phase space $\mathfrak{X}_{0}$ is a linear space of functions mapping $B$ into $\mathbb{R}$, we consider the following mixed problem:

$$
\begin{aligned}
D_{t} z(t, x) & =\sum_{i=1}^{n} f_{i}\left(t, x, z_{(t, x)}\right) D_{x_{i}} z(t, x)+h\left(t, x, z_{(t, x)}\right), \\
z(t, x) & =\phi(t, x), \quad(t, x) \in E_{0}^{*} \cup \partial_{0} E_{a} .
\end{aligned}
$$

In this paper we consider Carathéodory solutions of (1),(2) local with respect to the first variable. In other words an absolutely continuous function

1991 Mathematics Subject Classification: 35F30, 35L60, 35R10.

Key words and phrases: functional differential equation, unbounded delay, mixed problem, Carathéodory solutions, bicharacteristics, fixed-point theorem. 
$z: E_{\bar{a}}^{*} \rightarrow \mathbb{R}$ is said to be a solution of (1), (2) if it satisfies equation (1) almost everywhere on $E_{\bar{a}}$ and fulfills the initial-boundary condition (2) on $E_{0}^{*} \cup \partial_{0} E_{\bar{a}}$ for a certain $\bar{a} \in(0, a]$.

We prove the existence and uniqueness theorem for the mixed problem (1), (2) in which the differential equation has unbounded delay. This paper initiates studying mixed problems for such equations. Mixed problems for quasilinear functional differential equations with bounded delay have been studied by Kamont and Topolski [9] and Turo [11] for Carathéodory solutions and by Człapiński [3] for classical solutions. Analogous results for nonlinear equations have been given in [5]. Differential or differencedifferential inequalities and the Chaplygin method for first order functional mixed problems have been studied in [1], [4] while difference-differential equations in [7].

The theory of ordinary functional differential equations with unbounded delay has rich literature including two monographs by Hino, Murakami and Naito [6] and Lakshmikantham, Wen and Zhang [10]. In this paper we adapt the axiomatic approach of [6] to the mixed problem (1),(2). This approach has already been used for studying partial functional differential equations: in [8] to initial value problem for first order quasilinear equations and in [2] to the Darboux problem for equations of the second order.

If $z: E_{\bar{a}}^{*} \rightarrow \mathbb{R}$, where $\bar{a} \in(0, a]$, then for any $(t, x) \in E_{\bar{a}}$ we put

$$
\begin{aligned}
& \|z\|_{0}^{[t, x]}=\sup \{|z(\tau, s)|:(\tau, s) \in[0, t] \times[x, x+r]\}, \\
& \|z\|_{L}^{[t, x]}=\sup \left\{\frac{|z(\tau, s)-z(\tau, \bar{s})|}{|s-\bar{s}|}:\right. \\
& \qquad(\tau, s),(\tau, \bar{s}) \in[0, t] \times[x, x+r], s \neq \bar{s}\} .
\end{aligned}
$$

Throughout this paper we assume that spaces $\mathfrak{X}_{0}, \mathfrak{X}_{L}$ satisfy the following:

Assumption $\mathrm{H}_{1}$. We assume that $\mathfrak{X}_{0}$ is a Banach space with a norm $\|\cdot\|_{\mathfrak{X}_{0}}$ with the following properties:

If $z: E_{\bar{a}}^{*} \rightarrow \mathbb{R}, \bar{a} \in(0, a]$, is such that $z_{(0, s)} \in \mathfrak{X}_{0}$ for $s \in[-b, b]$ and $z$ is continuous on $E_{\bar{a}}$ then

(i) for any fixed $(t, x) \in E_{\bar{a}}$ we have $z_{(t, x)} \in \mathfrak{X}_{0}$ and

$$
\left\|z_{(t, x)}\right\|{\mathfrak{X}_{0}} \leq K_{0}\|z\|_{0}^{[t, x]}+M_{0}\left\|z_{(0, x)}\right\| \|_{\mathfrak{X}_{0}},
$$

where $K_{0}, M_{0} \in \mathbb{R}_{+}$are constants independent of $z$;

(ii) the function $E_{\bar{a}} \ni(t, x) \mapsto z_{(t, x)} \in \mathfrak{X}_{0}$ is continuous.

We assume furthermore that there is a subspace $\mathfrak{X}_{L}$ of $\mathfrak{X}_{0}$ which is also a Banach space with a norm $\|\cdot\|_{\mathfrak{X}_{L}}$ and has analogous properties: 
If $z: E_{\bar{a}}^{*} \rightarrow \mathbb{R}, \bar{a} \in(0, a]$, is such that $z_{(0, s)} \in \mathfrak{X}_{L}$ for $s \in[-b, b]$, and $z$ is continuous on $E_{\bar{a}}$ and Lipschitzean with respect to the second variable, then

(i) for any fixed $(t, x) \in E_{\bar{a}}$ we have $z_{(t, x)} \in \mathfrak{X}_{L}$ and

$$
\left\|z_{(t, x)}\right\|{\mathfrak{X}_{L}} \leq K_{L}\left(\|z\|_{0}^{[t, x]}+\|z\|_{L}^{[t, x]}\right)+M_{L}\left\|z_{(0, x)}\right\|_{\mathfrak{X}_{L}},
$$

where $K_{L}, M_{L} \in \mathbb{R}_{+}$are constants independent of $z$;

(ii) the function $E_{\bar{a}} \ni(t, x) \mapsto z_{(t, x)} \in \mathfrak{X}_{L}$ is continuous.

ExAmple 1. Let $\mathfrak{X}_{0}$ be the space of all functions $w: B \rightarrow \mathbb{R}$ such that $w$ is uniformly continuous and bounded on $B$, with the norm

$$
\|w\|_{\mathfrak{X}_{0}}=\sup \{|w(\tau, s)|:(\tau, s) \in B\} .
$$

Furthermore, let $\mathfrak{X}_{L}$ denote the space of all $w \in \mathfrak{X}_{0}$ such that

$$
\|w\|_{L}=\sup \left\{\frac{|w(\tau, s)-w(\tau, \bar{s})|}{|s-\bar{s}|}:(\tau, s),(\tau, \bar{s}) \in B, s \neq \bar{s}\right\}<\infty,
$$

with the norm $\|w\|_{\mathfrak{X}_{L}}=\|w\|_{\mathfrak{X}_{0}}+\|w\|_{L}$. Then Assumption $\mathrm{H}_{1}$ is satisfied with $K_{0}=M_{0}=K_{L}=M_{L}=1$.

More examples of spaces $\mathfrak{X}_{0}, \mathfrak{X}_{L}$ satisfying the above axioms are given at the end of Section 3.

Write

$$
\mathfrak{X}_{0}[p]=\left\{w \in \mathfrak{X}_{0}:\|w\|_{\mathfrak{X}_{0}} \leq p\right\}, \quad \mathfrak{X}_{L}[p]=\left\{w \in \mathfrak{X}_{L}:\|w\|_{\mathfrak{X}_{L}} \leq p\right\},
$$

where $p \in \mathbb{R}_{+}$.

Denote by $\Theta$ the set of all functions $\gamma:[0, a] \times \mathbb{R}_{+} \rightarrow \mathbb{R}_{+}$such that $\gamma(t, \cdot): \mathbb{R}_{+} \rightarrow \mathbb{R}_{+}$is nondecreasing for almost all $t \in[0, a]$ and $\gamma(\cdot, p)$ : $[0, a] \rightarrow \mathbb{R}_{+}$is Lebesgue integrable for all $p \in \mathbb{R}_{+}$.

\section{Bicharacteristics}

Assumption $\mathrm{H}_{2}$. Suppose that $\phi: E_{0}^{*} \cup \partial_{0} E_{a} \rightarrow \mathbb{R}$ is such that

(i) $\phi_{(0, s)} \in \mathfrak{X}_{L}$ for $s \in[-b, b]$, and there are constants $\lambda_{0}, \lambda_{L}, \lambda_{1} \in \mathbb{R}_{+}$ such that $\left\|\phi_{(0, s)}\right\|_{\mathfrak{X}_{0}} \leq \lambda_{0}$ and $\left\|\phi_{(0, s)}\right\|_{\mathfrak{X}_{L}} \leq \lambda_{L}$ for $s \in[-b, b]$, and

$$
\| \phi_{(0, s)}-\phi_{(0, \bar{s})}||_{\mathfrak{X}_{0}} \leq \lambda_{1}|s-\bar{s}| \quad \text { for } s, \bar{s} \in[-b, b] ;
$$

(ii) there are constants $l_{0}, l_{1}, \widetilde{l}_{1} \in \mathbb{R}_{+}$such that

$$
|\phi(t, x)| \leq l_{0}, \quad|\phi(t, x)-\phi(\bar{t}, \bar{x})| \leq \widetilde{l}_{1}|t-\bar{t}|+l_{1}|x-\bar{x}| \quad \text { on } \partial_{0} E_{a} .
$$

Suppose that Assumption $\mathrm{H}_{2}$ is satisfied and that we have constants $q=\left(q_{0}, q_{1}, \widetilde{q}_{1}\right) \in \mathbb{R}_{+}^{3}, q_{0} \geq l_{0}, q_{1} \geq l_{1}, \widetilde{q}_{1} \geq \widetilde{l}_{1}$. For any $\bar{a} \in(0, a]$ we denote 
by $\mathfrak{I}_{\bar{a} . \phi}[q]$ the set of all functions $z: E_{\bar{a}}^{*} \rightarrow \mathbb{R}$ such that

(i) $z(t, x)=\phi(t, x)$ on $E_{0}^{*} \cup \partial_{0} E_{\bar{a}}$;

(ii) $|z(t, x)| \leq q_{0}$ and $|z(t, x)-z(\bar{t}, \bar{x})| \leq \widetilde{q}_{1}|t-\bar{t}|+q_{1}|x-\bar{x}|$ on $E_{\bar{a}} \cup \partial_{0} E_{\bar{a}}$.

Assumption $\mathrm{H}_{3}$. Suppose that

(i) $f(\cdot, x, w):[0, a] \rightarrow \mathbb{R}^{n}$ is measurable for $(x, w) \in[-b, b] \times \mathfrak{X}_{0}$ and $f(t, \cdot):[-b, b] \times \mathfrak{X}_{0} \rightarrow \mathbb{R}^{n}$ is continuous for almost all $t \in[0, a]$;

(ii) there are a nondecreasing function $\alpha_{0}: \mathbb{R}_{+} \rightarrow \mathbb{R}_{+}$and $\alpha_{1} \in \Theta$ such that for almost all $t \in[0, a]$ we have $|f(t, x, w)| \leq \alpha_{0}(p)$, where $(x, w) \in$ $[-b, b] \times \mathfrak{X}_{0}[p], p \in \mathbb{R}_{+}$, and

$$
|f(t, x, w)-f(t, \bar{x}, \bar{w})| \leq \alpha_{1}(t, p)\left[|x-\bar{x}|+\|w-\bar{w}\|_{\mathfrak{X}_{0}}\right]
$$

for $(x, w),(\bar{x}, \bar{w}) \in[-b, b] \times \mathfrak{X}_{L}[p], p \in \mathbb{R}_{+}$;

(iii) for every $q \in \mathbb{R}_{+}$there is $\delta(p)>0$ such that $f_{i}(t, x, w) \geq \delta(p)$, $i=1, \ldots, n$, for $(x, w) \in[-b, b] \times \mathfrak{X}_{0}[p], p \in \mathbb{R}_{+}$, and a.a. $t \in[0, a]$.

For a fixed $z \in \mathfrak{I}_{\bar{a} . \phi}[q]$, where $\bar{a} \in(0, a]$ and for any $(t, x) \in E_{\bar{a}}$, we consider the Cauchy problem

$$
\frac{d}{d \tau} \varrho(\tau)=-f\left(\tau, \varrho(\tau), z_{(\tau, \varrho(\tau))}\right), \quad \varrho(t)=x .
$$

If Assumption $\mathrm{H}_{3}$ holds then there is a unique solution $g[z](\cdot, t, x)=$ $\left(g_{1}[z](\cdot, t, x), \ldots, g_{n}[z](\cdot, t, x)\right)$ of problem $(4)$. Let $\lambda[z](t, x)$ be the left end of the maximal interval on which the solution $g[z](\cdot, t, x)$ is defined. Then $(\lambda[z](t, x), g[z](\lambda[z](t, x), t, x)) \in\left(E_{0}^{*} \cup \partial_{0} E_{\bar{a}}\right) \cap E_{\bar{a}}$ by Assumption $\mathrm{H}_{3}($ iii $)$ and we may define the following two sets:

$$
\begin{aligned}
& E_{\bar{a} 0}[z]=\left\{(t, x) \in E_{\bar{a}}: \lambda[z](t, x)=0\right\}, \\
& E_{\bar{a} b}[z]=\left\{(t, x) \in E_{\bar{a}}: g_{i}[z](\lambda[z](t, x), t, x)=b_{i} \text { for some } 1 \leq i \leq n\right\} .
\end{aligned}
$$

Lemma 1. Suppose that Assumptions $\mathrm{H}_{1}-\mathrm{H}_{3}$ are satisfied, $z, \bar{z} \in \mathfrak{I}_{\bar{a} . \phi}[q]$, where $\bar{a} \in(0, a]$, and $(t, x),(\bar{t}, \bar{x}) \in E_{\bar{a}}$. If the intervals $K_{1}=[\max \{\lambda[z](t, x)$, $\lambda[z](\bar{t}, \bar{x})\}, \min \{t, \bar{t}\}]$ and $K_{2}=[\max \{\lambda[z](t, x), \lambda[\bar{z}](t, x)\}, t]$ are nonempty then

$$
\begin{aligned}
& |g[z](\tau, t, x)-g[z](\tau, \bar{t}, \bar{x})| \\
& \quad \leq\left[|x-\bar{x}|+\alpha_{0}\left(d_{0}\right)|t-\bar{t}|\right] \exp \left[d_{1}\left|\int_{t}^{\tau} \alpha_{1}\left(\xi, d_{L}\right) d \xi\right|\right]
\end{aligned}
$$

for $t \in K_{1}$, where $d_{0}=K_{0} q_{0}+M_{0} \lambda_{0}, d_{1}=1+K_{0} q_{1}+M_{0} \lambda_{1}$, and

$$
\begin{aligned}
& |g[z](\tau, t, x)-g[\bar{z}](\tau, t, x)| \\
& \quad \leq\left|\int_{t}^{\tau} K_{0} \alpha_{1}\left(\xi, d_{L}\right)\|z-\bar{z}\|_{E_{\xi}} d \xi\right| \exp \left[d_{1}\left|\int_{t}^{\tau} \alpha_{1}\left(\xi, d_{L}\right) d \xi\right|\right]
\end{aligned}
$$


for $t \in K_{2}$, where $d_{L}=K_{L}\left(q_{0}+q_{1}\right)+M_{L} \lambda_{L}$ and $\|\cdot\|_{E_{\xi}}$ denotes the supremum norm on $E_{\xi}$.

Pro of. It follows from Assumptions $\mathrm{H}_{1}$ and $\mathrm{H}_{2}$ that $\left.\left\|z_{(\tau, \xi)}-z_{(\tau, \bar{\xi})}\right\|\right|_{\mathfrak{X}_{0}}$

$$
\begin{aligned}
& \left.\leq K_{0} \sup _{(s, \eta) \in[0, \tau] \times[\xi, \xi+r]}|z(s, \eta)-z(s, \bar{\xi}-\xi+\eta)|+M_{0} \| z_{(0, \xi)}-z_{(0, \bar{\xi})}\right) \|_{\mathfrak{X}_{0}} \\
& \leq\left[K_{0} q_{1}+M_{0} \lambda_{1}\right]|x-\bar{x}| \quad \text { for }(\tau, \xi),(\tau, \bar{\xi}) \in E_{\bar{a}} .
\end{aligned}
$$

This gives the following Lipschitz condition:

$$
\left|f\left(\tau, \xi, z_{(\tau, \xi)}\right)-f\left(\tau, \bar{\xi}, z_{(\tau, \bar{\xi})}\right)\right| \leq d_{1} \alpha_{1}\left(\tau, d_{L}\right)|\xi-\bar{\xi}|
$$

for the right-hand side of (4), which therefore fulfills the Carathéodory conditions. Thus the existence of the unique solution of (4) follows from classical theorems. Let $g=g[z]$ and $\bar{g}=g[\bar{z}]$. Transforming (4) into an integral form we get

$$
\begin{aligned}
& |g(\tau, t, x)-g(\tau, \bar{t}, \bar{x})| \\
& \leq|x-\bar{x}|+\left|\int_{\bar{t}}^{t} f\left(\xi, g(\xi, \bar{t}, \bar{x}), z_{(\xi, g(\xi, \bar{t}, \bar{x}))}\right) d \xi\right| \\
& \quad+\left|\int_{t}^{\tau}\left[f\left(\xi, g(\xi, t, x), z_{(\xi, g(\xi, t, x))}\right)-f\left(\xi, g(\xi, \bar{t}, \bar{x}), z_{(\xi, g(\xi, \bar{t}, \bar{x}))}\right)\right] d \xi\right| \\
& \leq|x-\bar{x}|+\alpha_{0}\left(d_{0}\right)|t-\bar{t}|+\left|\int_{t}^{\tau} d_{1} \alpha_{1}\left(\xi, d_{L}\right)\right| g(\xi, t, x)-g(\xi, \bar{t}, \bar{x})|d \xi|,
\end{aligned}
$$

from which we get (5) by the Gronwall lemma.

Analogously we get

$$
\begin{aligned}
& |g(\tau, t, x)-\bar{g}(\tau, t, x)| \\
& \leq\left|\int_{t}^{\tau}\left[f\left(\xi, g(\xi, t, x), z_{(\xi, g(\xi, t, x))}\right)-f\left(\xi, \bar{g}(\xi, t, x), \bar{z}_{(\xi, \bar{g}(\xi, t, x))}\right)\right] d \xi\right| \\
& \leq\left|\int_{t}^{\tau} K_{0} \alpha_{1}\left(\xi, d_{L}\right)\|z-\bar{z}\|_{E_{\xi}} d \xi\right| \\
& \quad+\left|\int_{t}^{\tau} d_{1} \alpha_{1}\left(\xi, d_{L}\right)\right| g(\xi, t, x)-\bar{g}(\xi, t, x)|d \xi|
\end{aligned}
$$

and (6) using the Gronwall lemma again. This completes the proof of Lemma 1. 
Lemma 2. If Assumptions $\mathrm{H}_{1}-\mathrm{H}_{3}$ are satisfied and $z, \bar{z} \in \mathfrak{I}_{\bar{a} . \phi}[q]$ then

$$
\begin{aligned}
\mid \lambda[z](t, x)- & \lambda[z](\bar{t}, \bar{x}) \mid \\
\leq & {\left[|x-\bar{x}|+\alpha_{0}\left(d_{0}\right)|t-\bar{t}|\right] \frac{1}{\delta\left(d_{0}\right)} \exp \left[d_{1} \int_{0}^{t} \alpha_{1}\left(\xi, d_{L}\right) d \xi\right] }
\end{aligned}
$$

and

$$
\begin{aligned}
\mid \lambda[z](t, x) & -\lambda[\bar{z}](t, x) \mid \\
\leq & \frac{1}{\delta\left(d_{0}\right)} \int_{0}^{t} K_{0} \alpha_{1}\left(\xi, d_{L}\right) d \xi \exp \left[d_{1} \int_{0}^{t} \alpha_{1}\left(\xi, d_{L}\right) d \xi\right]\|z-\bar{z}\|_{E_{\bar{a}}},
\end{aligned}
$$

for $(x, y) \in E_{\bar{a}}$.

Proof. Write $\lambda=\lambda[z], g=g[z]$ and $\bar{\lambda}=\lambda[\bar{z}], \bar{g}=g[\bar{z}]$. Since (7) is obviously satisfied if $(t, x),(\bar{t}, \bar{x}) \in E_{\bar{a} 0}[z]$, without loss of generality we may assume that $\lambda(\bar{t}, \bar{x}) \leq \lambda(t, x)$ and $(t, x) \in E_{\bar{a} b}[z]$. Let $1 \leq i \leq n$ be such that $g_{i}(\lambda(t, x), t, x)=b_{i}$. Then

$$
\begin{aligned}
g_{i}(\lambda(t, x), t, x)-g_{i}(\lambda(t, x), \bar{t}, \bar{x}) & \geq g_{i}(\lambda(\bar{t}, \bar{x}), \bar{t}, \bar{x})-g_{i}(\lambda(t, x), \bar{t}, \bar{x}) \\
& =\int_{\lambda(t, \bar{t}, \bar{x})}^{\lambda(x)} f_{i}\left(\tau, g(\tau, \bar{t}, \bar{x}), z_{(\tau, g(\tau, \bar{t}, \bar{x}))}\right) d \tau \\
& \geq \delta\left(d_{0}\right)[\lambda(t, x)-\lambda(\bar{t}, \bar{x})] .
\end{aligned}
$$

Together with (5) this yields (7).

Also (8) is obviously satisfied if $(t, x) \in E_{\bar{a} 0}[z] \cap E_{\bar{a} 0}[\bar{z}]$, so we may assume that $\bar{\lambda}(t, x) \leq \lambda(t, x)$ and $(t, x) \in E_{\bar{a} b}[z]$. Analogously we prove

$$
g_{i}(\lambda(t, x), t, x)-\bar{g}_{i}(\lambda(t, x), t, x) \geq \delta\left(d_{0}\right)[\lambda(t, x)-\bar{\lambda}(t, x)],
$$

which together with (6) gives (8). This completes the proof of Lemma 2.

3. The main result. Now we prove a theorem on existence and uniqueness of solutions of the mixed problem (1), (2).

Assumption $\mathrm{H}_{4}$. Suppose that

(i) $h(\cdot, x, w):[0, a] \rightarrow \mathbb{R}$ is measurable for $(x, w) \in[-b, b] \times \mathfrak{X}_{0}$ and $h(t, \cdot):[-b, b] \times \mathfrak{X}_{0} \rightarrow \mathbb{R}$ is continuous for almost all $t \in[0, a] ;$

(ii) there are a nondecreasing function $\beta_{0}: \mathbb{R}_{+} \rightarrow \mathbb{R}_{+}$and $\beta_{1} \in \Theta$ such that for almost all $t \in[0, a]$ we have $|h(t, x, w)| \leq \beta_{0}(p)$, where $(x, w) \in$ $[-b, b] \times \mathfrak{X}_{0}[p], p \in \mathbb{R}_{+}$, and

$$
|h(t, x, w)-h(t, \bar{x}, \bar{w})| \leq \beta_{1}(t, p)\left[|x-\bar{x}|+\|w-\bar{w}\|_{\mathfrak{X}_{0}}\right]
$$

for $(x, w),(\bar{x}, \bar{w}) \in[-b, b] \times \mathfrak{X}_{L}[p], p \in \mathbb{R}_{+}$. 
We are now in a position to explain why the Lipschitz conditions for $f$ and $h$ are not uniform with respect to $w$. Given $\widetilde{f}=\left(\widetilde{f}_{1}, \ldots, \widetilde{f}_{n}\right): E_{a} \times \mathbb{R}$ $\rightarrow \mathbb{R}^{n}$ and $\widetilde{h}: E_{a} \times \mathbb{R} \rightarrow \mathbb{R}$ consider the following equation with deviated argument:

$$
\begin{aligned}
D_{t} z(t, x)+\sum_{i=1}^{n} \widetilde{f}_{i}\left(t, x, z\left(\psi_{0}(t), \psi(t, x)\right)\right) D_{x_{i}} z( & t, x) \\
& =\widetilde{h}\left(t, x, z\left(\psi_{0}(t), \psi(t, x)\right)\right),
\end{aligned}
$$

where $\psi_{0}:[0, a] \rightarrow \mathbb{R}, \psi: E_{a} \rightarrow \mathbb{R}^{n}, \psi(t) \leq t$ and $\psi(t, x)-x \in[0, r]$ for $(t, x) \in E_{a}$. We expect that the above equation can be obtained as a special case of (1) under suitable assumptions.

Suppose that there are a Lebesgue integrable function $\widetilde{\alpha}_{1}:[0, a] \rightarrow \mathbb{R}_{+}$ and a constant $A \in \mathbb{R}_{+}$such that

$$
\begin{aligned}
|\widetilde{f}(t, x, z)-\widetilde{f}(t, \bar{x}, \bar{z})| & \leq \widetilde{\alpha}_{1}(t)[|x-\bar{x}|+|z-\bar{z}|], \\
|\psi(t, x)-\psi(t, \bar{x})| & \leq A|x-\bar{x}|,
\end{aligned}
$$

for $(t, x),(\bar{t}, \bar{x}) \in E_{a} \times \mathfrak{X}_{0}, z, \bar{z} \in \mathbb{R}$. We define

$$
\left.f(t, x, w)=\widetilde{f}\left(t, x, w\left(\psi_{0}(t)-t, \psi(t, x)-x\right)\right)\right), \quad(t, x, w) \in E_{a} \times \mathfrak{X}_{0} .
$$

with $\mathfrak{X}_{0}, \mathfrak{X}_{L}$ as in Example 1. We show that even though the Lipschitz condition for $\widetilde{f}$ is uniform it is not the case for $f$. Similar considerations apply to $h$.

For $(t, x),(t, \bar{x}) \in E_{a}$ and $w, \bar{w} \in \mathfrak{X}_{L}[p], p \in \mathbb{R}_{+}$, we have

$$
\begin{aligned}
|f(t, x, w)-f(t, \bar{x}, \bar{w})| \leq & \widetilde{\alpha}_{1}(t)\left[|x-\bar{x}|+\mid w\left(\psi_{0}(t)-t, \psi(t, x)-x\right)\right. \\
& \left.-\bar{w}\left(\psi_{0}(t)-t, \psi(t, \bar{x})-\bar{x}\right) \mid\right] \\
\leq & \widetilde{\alpha}_{1}(t)[1+p(1+A)]|x-\bar{x}|+\widetilde{\alpha}_{1}(t)\|w-\bar{w}\|_{\mathfrak{X}_{0}} .
\end{aligned}
$$

Consequently, $f$ satisfies the Lipschitz condition of Assumption $\mathrm{H}_{3}$ with $\alpha_{1}(t, p)=\widetilde{\alpha}_{1}(t)[1+p(1+A)]$, which obviously is not uniform.

REMARK 1. A class of differential-integral equations can also be obtained from (1) by specializing $f$ and $h$.

We define the operator $\mathcal{W}$ on $\mathfrak{I}_{\bar{a} . \phi}[q]$ by

(9) $\quad(\mathcal{W} z)(t, x)$

$$
=\left\{\begin{array}{c}
\phi(\lambda[z](t, x), g[z](\lambda[z](t, x), t, x)) \\
+\int_{x}^{\lambda[z](t, x)} h\left(\tau, g[z](\tau, t, x), z_{(\tau, g[z](\tau, t, x)}\right) d \tau \quad \text { for }(t, x) \in E_{\bar{a}}, \\
\phi(t, x) \quad \text { for }(t, x) \in E_{0}^{*} \cup \partial_{0} E_{\bar{a}} .
\end{array}\right.
$$


REMARK 2. The right-hand side of (9) arises in the following way. We consider (1) along bicharacteristics

$$
\begin{aligned}
& D_{t} z(\tau, g[z](\tau, t, x)) \\
& \quad-\sum_{i=1}^{n} f_{i}\left(\tau, g[z](\tau, t, x), z_{(\tau, g[z](\tau, t, x))}\right) D_{x_{i}} z(t, g[z](\tau, t, x)) \\
& =h\left(\tau, g[z](\tau, t, x), z_{(\tau, g[z](\tau, t, x))}\right),
\end{aligned}
$$

from which by (4) we get

$$
\frac{d}{d \tau} z(\tau, g[z](\tau, t, x))=h\left(\tau, g[z](\tau, t, x), z_{(\tau, g[z](\tau, t, x))}\right) .
$$

Integrating this equation with respect to $\tau$ on the interval $[\lambda[z](t, x), t]$ we get the right-hand side of (9).

Define the constants

$$
\begin{aligned}
S_{0 \bar{a}}= & l_{0}+\bar{a} \beta_{0}\left(d_{0}\right), \\
S_{1 \bar{a}}= & {\left[\left(\widetilde{l}_{1}+l_{1} \alpha_{0}\left(d_{0}\right)+\beta_{0}\left(d_{0}\right)\right) \frac{1}{\delta\left(d_{0}\right)}+l_{1}+\int_{0}^{\bar{a}} d_{1} \alpha_{1}\left(\tau, d_{L}\right) d \tau\right] } \\
& \times \exp \left[d_{1} \int_{0}^{\bar{a}} \alpha_{1}\left(\xi, d_{L}\right) d \xi\right] .
\end{aligned}
$$

TheOREM 3. If Assumptions $\mathrm{H}_{1}-\mathrm{H}_{4}$ are satisfied, then there are constants $q_{0}, q_{1}, \widetilde{q}_{1} \in \mathbb{R}_{+}$such that for $\bar{a} \in(0, a]$ sufficiently small the operator $\mathcal{W}$ maps $\mathfrak{I}_{\bar{a} . \phi}[q]$ into itself.

Proof. Let $q_{0}>l_{0}$,

$$
\begin{aligned}
& \widetilde{q}_{1}>\max \left\{\tilde{l}_{1}, \beta_{0}\left(d_{0}\right)+\left(\widetilde{l}_{1}+l_{1} \alpha_{0}\left(d_{0}\right)+\beta_{0}\left(d_{0}\right)\right) \frac{\alpha_{0}\left(d_{0}\right)}{\delta\left(d_{0}\right)}\right\}, \\
& q_{1}>\left(\widetilde{l}_{1}+l_{1} \alpha_{0}\left(d_{0}\right)+\beta_{0}\left(d_{0}\right)\right) \frac{1}{\delta\left(d_{0}\right)}+l_{1}
\end{aligned}
$$

and $z \in \mathfrak{I}_{\bar{a} . \phi}[q]$. As in the proof of Lemma 2 we write $\lambda, g$ instead of $\lambda[z], g[z]$ for simplicity.

It is obvious that $\mathcal{W} z$ is continuous on $E_{\bar{a}}^{*}$. We prove that for sufficiently small $\bar{a} \in(0, a]$ we have

$$
\begin{aligned}
& |(\mathcal{W} z)(t, x)| \leq q_{0}, \\
& |(\mathcal{W} z)(t, x)-(\mathcal{W} z)(\bar{t}, \bar{x})| \leq \widetilde{q}_{1}|t-\bar{t}|+q_{1}|x-\bar{x}| \quad \text { on } E_{\bar{a}} \cup \partial_{0} E_{\bar{a}} .
\end{aligned}
$$

For all $(t, x) \in E_{\bar{a} b}[z]$ we have

$$
|(\mathcal{W} z)(t, x)| \leq l_{0}+\int_{\lambda(t, x)}^{t} \beta_{0}\left(d_{0}\right) d \xi \leq S_{0 \bar{a}}
$$


We also have

$$
\begin{aligned}
& |(\mathcal{W} z)(t, x)-(\mathcal{W} z)(\bar{t}, \bar{x})| \\
& \leq \widetilde{l}_{1}|\lambda(t, x)-\lambda(\bar{t}, \bar{x})|+l_{1}|g(\lambda(t, x), t, x)-g(\lambda(\bar{t}, \bar{x}), \bar{t}, \bar{x})| \\
& \quad+\beta_{0}\left(d_{0}\right)|t-\bar{t}|+\beta_{0}\left(d_{0}\right)|\lambda(t, x)-\lambda(\bar{t}, \bar{x})| \\
& +\int_{\lambda(t, x)}^{t} \beta_{1}\left(\tau, d_{L}\right)\left[|g(\tau, t, x)-g(\tau, \bar{t}, \bar{x})|+\left\|z_{(\tau, g(\tau, t, x))}-z_{(\tau, g(\tau, \bar{t}, \bar{x}))}\right\|_{\mathfrak{X}_{0}}\right] d \tau
\end{aligned}
$$

on $E_{\bar{a} b}[z]$, from which by (5) and (7) we get

(12) $|(\mathcal{W} z)(t, x)-(\mathcal{W} z)(\bar{t}, \bar{x})|$

$$
\leq\left[\beta_{0}\left(d_{0}\right)+S_{1 \bar{a}} \alpha_{0}\left(d_{0}\right)\right]|t-\bar{t}|+S_{1 \bar{a}}|x-\bar{x}| .
$$

Note that since the integral $\int_{\lambda(t, x)}^{t}$ is estimated by $\int_{0}^{\bar{a}}$ the estimates (11) and (12) will still be valid on $E_{\bar{a} 0}[z]$. Taking $\bar{a}$ sufficiently small in order that $S_{0 \bar{a}} \leq q_{0}, S_{1 \bar{a}} \leq q_{1}$ and $\beta_{0}\left(d_{0}\right)+S_{1 \bar{a}} \alpha_{0}\left(d_{0}\right) \leq \widetilde{q}_{1}$ we get (10) for all $(t, x) \in E_{\bar{a}}$. Since $l_{0}<q_{0}, l_{1}<q_{1}, \widetilde{l}_{1}<\widetilde{q}_{1}$ we see that (10) holds true also for $(t, x) \in \partial_{0} E_{\bar{a}}$.

THEOREM 4. If Assumptions $\mathrm{H}_{1}-\mathrm{H}_{4}$ are satisfied then there are constants $q_{0}, q_{1}, \widetilde{q}_{1} \in \mathbb{R}_{+}$such that problem (1),(2) has a unique solution on $E_{\bar{a}}$ in the class $\mathfrak{I}_{\bar{a} . \phi}[q]$ for sufficiently small $\bar{a} \in(0, a]$.

Proof. Let $q_{0}, q_{1}, \widetilde{q}_{1}$ be as in Theorem 3 . We prove that the operator $\mathcal{W}: \mathfrak{I}_{\bar{a} . \phi}[q] \rightarrow \mathfrak{I}_{\bar{a} . \phi}[q]$ is a contraction for sufficiently small $\bar{a} \in(0, a]$. Indeed, if $z, \bar{z} \in \mathfrak{I}_{\bar{a} . \phi}[q], g=g[z], \bar{g}=g[\bar{z}], \lambda=\lambda[z], \bar{\lambda}=\lambda[\bar{z}]$ then

$$
\begin{aligned}
& |(\mathcal{W} z)(t, x)-(\mathcal{W} \bar{z})(t, x)| \\
& \leq \widetilde{l}_{1}|\lambda(t, x)-\bar{\lambda}(t, x)|+l_{1}|g(\lambda(t, x), t, x)-\bar{g}(\bar{\lambda}(t, x), t, x)| \\
& \quad+\beta_{0}\left(d_{0}\right)|\lambda(t, x)-\bar{\lambda}(t, x)|
\end{aligned}
$$

from which by (6) and (8) we obtain

$$
\|\mathcal{W} z-\mathcal{W} \bar{z}\|_{E_{\bar{a}}} \leq S_{\bar{a}}\|z-\bar{z}\|_{E_{\bar{a}}}
$$

where $S_{\bar{a}}=\int_{0}^{\bar{a}} K_{0}\left[\alpha_{1}\left(\xi, d_{L}\right) S_{1 \bar{a}}+\beta_{1}\left(\xi, d_{L}\right)\right] d \xi$. Since $\lim _{\bar{a} \rightarrow 0^{+}} S_{\bar{a}}=0$ we may choose $\bar{a} \in(0, a]$ sufficiently small in order that $S_{\bar{a}}<1$. Consequently, $\mathcal{W}$ is a contraction with the metric $\|z-\bar{z}\|_{E_{\bar{a}}}$ and by the Banach theorem there exists a unique fixed point of $\mathcal{W}$. Denoting it by $z^{*}$ we prove that it is a solution of (1). 
For any $(t, x) \in E_{\bar{a} 0}\left[z^{*}\right]$ we have

$$
z^{*}(t, x)=\phi(0, g(0, t, x))+\int_{0}^{t} h\left(\tau, g(\tau, t, x), z_{(\tau, g(\tau, t, x))}^{*}\right) d \tau .
$$

For fixed $t$ we consider the transformation $x \mapsto g(0, t, x)=\xi$. Upon using this transformation and the group property $g(\tau, t, x)=g(\tau, 0, \xi)$ the relation (13) takes the form

$$
z^{*}(t, g(t, 0, \xi))=\phi(0, \xi)+\int_{0}^{t} h\left(\tau, g(\tau, 0, \xi), z_{(\tau, g(\tau, 0, \xi))}^{*}\right) d \tau .
$$

Differentiating this equation with respect to $t$ we get

$$
\begin{aligned}
D_{t} z^{*}(t, g(t, 0, \xi))+\sum_{i=1}^{n} D_{x_{i}} z^{*}(t, g(t, 0, \xi)) & \frac{d g_{i}}{d \tau}(t, 0, \xi) \\
& =h\left(t, g(t, 0, \xi), z_{(t, g(t, 0, \xi))}^{*}\right) .
\end{aligned}
$$

Making use of (4) and the inverse transformation $\xi \mapsto g(t, 0, \xi)=x$ which preserves sets of measure zero we get (1) almost everywhere on $E_{\bar{a} 0}\left[z^{*}\right]$.

For any $(t, x) \in E_{\bar{a} b}\left[z^{*}\right]$ we have

$$
\begin{aligned}
z^{*}(t, x)= & \phi(\lambda(t, x), g(\lambda(t, x), t, x)) \\
& +\int_{\lambda(t, x)}^{t} h\left(\tau, g(\tau, t, x), z_{(\tau, g(\tau, t, x))}^{*}\right) d \tau .
\end{aligned}
$$

For simplicity of notation suppose that $g_{i}(\lambda(t, x), t, x)=b_{i}$ for $i=n$ and write $\xi^{\prime}=\left(\xi_{1}, \ldots, \xi_{n-1}\right), g^{\prime}=\left(g_{1}, \ldots, g_{n-1}\right)$. Fixing $t$ and using the transformation $x \mapsto\left(g^{\prime}(\lambda(t, x), t, x), \lambda(t, x)\right)=\left(\xi^{\prime}, \eta\right)$ we see that (14) takes the form

$$
\begin{aligned}
z^{*}\left(t, g\left(t, \eta, \xi^{\prime}, b_{n}\right)\right)= & \phi\left(\eta, \xi^{\prime}, b_{n}\right) \\
& +\int_{\eta}^{t} h\left(\tau, g\left(\tau, \eta, \xi^{\prime}, b_{n}\right), z_{\left(\tau, g\left(\tau, \eta, \xi^{\prime}, b_{n}\right)\right)}^{*}\right) d \tau .
\end{aligned}
$$

Differentiating the above equation with respect to $t$ and, as previously, making use of the inverse transformation $\left(\xi^{\prime}, \eta\right) \mapsto g\left(t, \eta, \xi^{\prime}, b_{n}\right)=x$ and (4) we get (1) almost everywhere on $E_{\bar{a} b}\left[z^{*}\right]$. Since $z^{*} \in \mathfrak{I}_{\bar{a} . \phi}[q]$ obviously fulfills the mixed condition (2) this completes the proof of Theorem 2.

The most natural example of spaces $\mathfrak{X}_{0}, \mathfrak{X}_{L}$ satisfying Assumption $\mathrm{H}_{1}$ was given in Section 1. Note that if we replace the uniform continuity in Example 1 by the continuity and existence of the $\operatorname{limit}_{t \rightarrow-\infty} w(t, x)$ uniformly with respect to $x \in[0, r]$ then we get another example of such spaces. This example includes as a special case spaces with bounded delay. Indeed, the space of all continuous functions $w:\left[-r_{0}, 0\right] \times[0, r] \rightarrow \mathbb{R}$ with 
the supremum norm is isometric to the space of all continuous functions $w: B \rightarrow \mathbb{R}$ such that for each $x \in[0, r]$ the function $w(\cdot, x)$ is constant on $\left(-\infty,-r_{0}\right]$. Analogous considerations apply to the space of functions which are additionally Lipschitzean on $\left[-r_{0}, 0\right] \times[0, r]$.

EXAMPLE 2 . Let $\gamma: \mathbb{R}_{-} \rightarrow(0, \infty)$ be continuous and nonincreasing. We define $\mathfrak{X}_{0}$ as the space of all functions $w: B \rightarrow \mathbb{R}$ such that

$$
\lim _{t \rightarrow-\infty} w(t, x) / \gamma(t)=0 \quad \text { for } x \in[0, r],
$$

with the norm

$$
\|w\|_{\mathfrak{X}_{0}}=\sup \{|w(\tau, s)| / \gamma(\tau):(\tau, s) \in B\} .
$$

Furthermore, let $\mathfrak{X}_{L}$ denote the space of all $w \in \mathfrak{X}_{0}$ such that

$$
\|w\|_{\gamma, L}=\sup \left\{\frac{|w(\tau, s)-w(\tau, \bar{s})|}{\gamma(\tau)|s-\bar{s}|}:(\tau, s),(\tau, \bar{s}) \in B, s \neq \bar{s}\right\}<\infty,
$$

with the norm $\|w\|_{\mathfrak{X}_{L}}=\|w\|_{\mathfrak{X}_{0}}+\|w\|_{\gamma, L}$. Then Assumption $\mathrm{H}_{1}$ is satisfied with $K_{0}=K_{L}=1 / \gamma(0), M_{0}=M_{L}=1$.

EXAmple 3 . Let $d \in \mathbb{R}_{+}, p \geq 1$ and let $\mathfrak{X}_{0}$ be the space of all functions $w: B \rightarrow \mathbb{R}$ such that

(i) $w$ is continuous and bounded on $(-d, 0] \times[0, r]$,

(ii) $\int_{-\infty}^{-d}|w(\tau, x)|^{p} d \tau<\infty$ for $x \in[0, r]$,

(iii) $w(t, \cdot):[0, r] \rightarrow \mathbb{R}$ is continuous for $t \in(-\infty,-d]$.

We endow $\mathfrak{X}_{0}$ with the norm

$$
\begin{aligned}
\|w\|_{\mathfrak{X}_{0}}= & \sup \{|w(\tau, s)|:(\tau, s) \in[-d, 0] \times[0, r]\} \\
& +\sup \left\{\left(\int_{-\infty}^{-d}|w(\tau, x)|^{p} d \tau\right)^{1 / p}: x \in[0, r]\right\} .
\end{aligned}
$$

For $\mathfrak{X}_{L}$ we take the space of all $w \in \mathfrak{X}_{0}$ such that condition (3) holds with the norm $\|w\|_{\mathfrak{X}_{L}}=\|w\|_{\mathfrak{X}_{0}}+\|w\|_{L}$. Then Assumption $\mathrm{H}_{1}$ is satisfied with

$$
K_{0}=K_{L}=1+(a-d)^{1 / p}, \quad M_{0}=M_{L}=1+d^{1 / p} .
$$

\section{References}

[1] P. Brandi, Z. Kamont and A. Salvadori, Differential and differential-difference inequalities related to mixed problems for first order partial differential-functional equations, Atti. Sem. Mat. Fis. Univ. Modena 39 (1991), 255-276.

[2] T. Człapiński, Existence of solutions of the Darboux problem for partial differential-functional equations with infinite delay in a Banach space, Comm. Math. 35 (1995), 111-122. 
[3] T. Człapiński, On the mixed problem for quasilinear differential-functional equations of the first order, Z. Anal. Anwendungen 16 (1997), 463-478.

[4] - On the Chaplyghin method for partial differential-functional equations of the first order, Univ. Iagel. Acta Math. 35 (1997), 137-149.

[5] -, On the mixed problem for hyperbolic partial differential-functional equations of the first order, Czechoslovak Math. J., to appear.

[6] Y. Hino, S. Murakami and T. Naito, Functional Differential Equations with Infinite Delay, Springer, 1991.

[7] G. A. Kamenskii and A. D. Myshkis, On the mixed type functional-differential equations, Nonlinear Anal. 30 (1997), 2577-2584.

[8] Z. Kamont, Hyperbolic functional differential equations with unbounded delay, Z. Anal. Anwendungen 18 (1999), 97-109.

[9] Z. Kamont and K. Topolski, Mixed problems for quasilinear hyperbolic differential-functional systems, Math. Balkanica 6 (1992), 313-324.

[10] V. Lakshmikantham, L. Wen and B. Zhang, Theory of Differential Equations with Unbounded Delay, Kluwer, 1994.

[11] J. Turo, Local generalized solutions of mixed problems for quasilinear hyperbolic systems of functional partial differential equations in two independent variables, Ann. Polon. Math. 49 (1989), 256-278.

Institute of Mathematics

University of Gdańsk

Wita Stwosza 57

80-952 Gdańsk, Poland

E-mail:czltsz@ksinet.univ.gda.pl 